\title{
MARKOV AND ARTIN NORMAL FORM THEOREM FOR BRAID GROUPS
}

\author{
L. A. BOKUT*, V.V. CHAYNIKOV*, AND K.P. SHUM**
}

\begin{abstract}
In this paper we will present the results of ArtinMarkov on braid groups by using the Gröbner-Shirshov basis. As a consequence we can reobtain the normal form of Artin-MarkovIvanovsky as an easy corollary.
\end{abstract}

\section{INTRODUCTION}

The theory of braid groups were first studied by E. Artin dated back to 1925 (see Ar25]). Artin established generators and defining relations of the braid group and a faithful representation theorem of the braid group as a subgroup of the automorphism group of a free group. Later on, Markov [Mar45] (1945) and Artin [Ar47] (1947) enriched the Artin generators by the Burau elements ( Bur32]) and find a presentation of the braid group in the Artin-Burau generators. We call it the Artin-Markov presentation of the braid group in the ArtinBurau generators. The main result of ( Mar45]) and ( [Ar47]) is the normal form theorem for the braid group. We call it the Artin-MarkovIvanovsky normal form theorem for Markov credited the result to A. Ivanovsky; it looks that there is no paper published by A. Ivanovskii himself. However, the proof of the normal form theorem of the braid group given by Markov and Artin are rather complicated because the automorphism of the free groups are involved. In this note we will try to escape the technical details of the proof given by Markov Mar45 and Artin Ar25. We will show by direct calculations (of compositions) that the Artin-Markov presentation of the braid group is the minimal Gröbner-Shirshov basis of the braid group in the Artin-Burau generators and under an appropriate ordering of group words, namely, the inverse tower ordering. As a consequence, by the Composition-Diamond Lemma the Artin-Markov-Ivanovsky normal form in the braid group follows as an immediate corollary of our result.

\section{BASIC NOTATIONS AND RESUlts}

In this section we give some basic notations and cite some useful results in the literature. We first let $\boldsymbol{X}$ be a linearly ordered set and

\footnotetext{
*Supported in part by the Russia's Fund for Fundamental Research and the Leading Scientific Schools Fund (Russia).

**Partially supported by a RGC(HK) grant 2003/04. 
$k$ a field. Let $k\langle\boldsymbol{X}\rangle$ the free associative algebra over $\boldsymbol{X}$ and $k$. On the set $\boldsymbol{X}^{*}$ of words generated by $\boldsymbol{X}$ we impose a well order $\leq$ compatible with the multiplication of words. We call this kind of order a monomial order.

Now, let $f \in k\langle\boldsymbol{X}\rangle$ be a polynomial with leading word $\bar{f}$. We say that $f$ is monic if $\bar{f}$ occurs in $f$ with coefficient 1 . We now formulate the following definitions.

Definition 2.1. Let $f$ and $g$ be two monic polynomials.

(i) Let $w$ be a word such that $w=\bar{f} b=a \bar{g}$, with $\operatorname{deg}(\bar{f})+\operatorname{deg}(\bar{g})>$ $\operatorname{deg}(w)$. Then we call the polynomial $(f, g)_{w}$ the intersection composition of $f$ and $g$ with respect to $w$ if $(f, g)_{w}=f b-a g$.

(ii) Let $w=\bar{f}=a \bar{g} b$. Then we call the polynomial $(f, g)_{w}=f-a g b$ the inclusion composition of $f$ and $g$ with respect to $w$.

In the above case, the transformation $f \mapsto(f, g)_{w}=f-a g b$ is called the elimination of the leading word (ELW) of $g$ in $f$.

(iii) Let $\boldsymbol{S} \subset k\langle\boldsymbol{X}\rangle$. We call a composition $(f, g)_{w}$ trivial relative to $\boldsymbol{S}$ (and $w$ ) if

$$
(f, g)_{w}=\sum \alpha_{i} a_{i} t_{i} b_{i}
$$

where $t_{i} \in \boldsymbol{S}, a_{i}, b_{i} \in \boldsymbol{X}^{*}$, and $a_{i} \bar{t}_{i} b_{i}<w$. In a notation, $(f, g)_{w} \equiv 0 \bmod (\boldsymbol{S}, w)$. In particular, if $(f, g)_{w}$ goes to zero by using ELW of polynomials from $\boldsymbol{S}$, then $(f, g)_{w}$ is trivial relative to $\boldsymbol{S}$. We assume that $f_{1}$ and $f_{2}$ are some polynomials satisfying the condition $f_{1} \equiv f_{2} \bmod (\boldsymbol{S}, w)$ if $f_{1}-f_{2} \equiv 0 \bmod (\boldsymbol{S}, w)$.

Definition 2.2. Then we call $\boldsymbol{S}$ a Gröbner-Shirshov set (basis) in $k\langle\boldsymbol{X}\rangle$ if any composition of polynomials from $\boldsymbol{S}$ is trivial relative to $\boldsymbol{S}$.

From now on, we denote the algebra with set of generators $\boldsymbol{X}$ and set of defining relations $\boldsymbol{S}$ by $\langle\boldsymbol{X} \mid \boldsymbol{S}\rangle$.

The following lemma and its applications to Gröbner-Shirshov bases was due to Newman [Ne42], Shirshov ( Sh62]), Buchberger [Bu65], Bu70] and Bergman [Be78]. The lemma given below was formulated by Bokut (see [Bo72], Bo76]).

Lemma 2.3 (Composition-Diamond Lemma). Let $R=\langle\boldsymbol{X} \mid \boldsymbol{S}\rangle$. The set of defining relations $\boldsymbol{S}$ is a Gröbner-Shirshov set if and only if the set

$$
\operatorname{Irr}(S)=\left\{u \in \boldsymbol{X}^{*} \mid u \neq a \bar{f} b, \text { for any } f \in \boldsymbol{S}\right\}
$$

of $\boldsymbol{S}$-irreducible words consists of a linear basis of $R$.

Definition 2.4. Let $\boldsymbol{S}$ be a Gröbner-Shirshov basis in $k\langle\boldsymbol{X}\rangle$. Then $\boldsymbol{S}$ is called a minimal Gröbner-Shirshov basis if for any $s \in \boldsymbol{S}, s$ is a linear combination of $\boldsymbol{S} \backslash\{s\}$-irreducible words. Any ideal of $k\langle\boldsymbol{X}\rangle$ has a unique minimal Gröbner-Shirshov basis (i.e., a set of generators of the ideal). 
If a subset $\boldsymbol{S}$ of $k\langle\boldsymbol{X}\rangle$ is not a Gröbner-Shirshov basis then one can add to $\boldsymbol{S}$ all nontrivial compositions of polynomials of $\boldsymbol{S}$, and continue this process (infinitely) many times in order to have a Gröbner-Shirshov set (basis) $\boldsymbol{S}^{\text {comp }}$ that generates the same ideal as $S$. This procedure is called the Buchberger - Shirshov algorithm [Sh62], Bu65], Bu70].

Definition 2.5. A polynomial $f$ is called semigroup relation if $f$ is of the form $u-v$, where $u, v \in \boldsymbol{X}^{*}$. If $S$ is a set of semigroup relations, then any nontrivial composition of $S$ has the same form. Consequently, the set $\boldsymbol{S}^{\text {comp }}$ also consists of semigroup relations.

Remark 2.6. Let $A=\operatorname{sgp}\langle\boldsymbol{X} \mid \boldsymbol{S}\rangle$ be a semigroup presentation. Then $\boldsymbol{S} \subset k\langle\boldsymbol{S}\rangle$ and one can find a Gröbner-Shirshov basis $\boldsymbol{S}^{\text {comp }}$. This set does not depend on $k$ and it consists of semigroup relations. In this case, we call $\boldsymbol{S}^{\text {comp }}$ a Gröbner-Shirshov basis of $A$. It is the same as a Gröbner-Shirshov basis of the semigroup algebra $k A=\langle\boldsymbol{X} \mid \boldsymbol{S}\rangle$.

We now introduce the concept of inverse tower ordering of words.

Definition 2.7. Let $X=Y \dot{U} Z$, words $Y^{*}$ and the letters $Z$ are well ordered. Suppose that the order on $Y^{*}$ is monomial. Any word in $X$ has the form $u=u_{0} z_{1} \ldots u_{k-1} z_{k} u_{k}$, where $k \geq 0, z_{i} \in Z, u_{i} \in Y^{*}$. Define the inverse weight of the word $u \in X$ by:

$$
\operatorname{inwt}(u)=\left(k, u_{k}, z_{k}, \ldots, z_{1}, u_{0}\right) .
$$

Now we order inverse weights lexicographically and define

$$
u>v \Longleftrightarrow \operatorname{inwt}(u)>\operatorname{inwt}(v) \text {. }
$$

Then we call the above order the inverse tower order. Clearly, the above order is a monomial order.

In case $Y=T \dot{U} U$ and $Y^{*}$ are endowed with the inverse tower order, we call order of words on $X$ the inverse tower order of word relative the presentation

$$
X=(T \bigcup U) \bigcup Z
$$

In general, we can define the inverse tower order of $X$-words relative to the presentation

$$
X=\left(\ldots\left(X^{(n)} \bigcup \dot{\bigcup} X^{(n-1)}\right) \dot{\bigcup} \ldots\right) \bigcup X^{(0)},
$$

where $X^{(n)}$-words are endowed by a monomial order.

Definition 2.8. Let $\Sigma=\left\{\sigma_{1}, \ldots, \sigma_{n-1}\right\}$ be a finite alphabet. Then, the following group presentation define the $n$-strand braid group:

$$
B_{n}=\left\langle\Sigma \mid \sigma_{i+1} \sigma_{i} \sigma_{i+1}=\sigma_{i} \sigma_{i+1} \sigma_{i}, \sigma_{i} \sigma_{j}=\sigma_{j} \sigma_{i}, i-j>1\right\rangle
$$

Here any index falls into the interval $[1, n-1]$.

In the braid group $B_{n}$, we now introduce a new set of generators. We call them the Artin-Burau generators. 
In the braid group $B_{n}$, we let

$$
s_{i, i+1}=\sigma_{i}^{2}, s_{i, j+1}=\sigma_{j} \ldots \sigma_{i+1} \sigma_{i}^{2} \sigma_{i+1}^{-1} \ldots \sigma_{j}^{-1},
$$

where $1 \leq i<j \leq n-1$.

Form the set

$$
S_{j}=\left\{s_{i, j}, s_{i, j}^{-1}, 2<i<j<n\right\}
$$

and

$$
\Sigma^{-1}=\left\{\sigma_{1}^{-1}, \ldots, \sigma_{n-1}^{-1}\right\} .
$$

Then the set

$$
S=S_{n} \cup S_{n-1} \cup \cdots \cup S_{2} \cup \Sigma^{-1}
$$

generates $B_{n}$ as a semigroup. We call elements of $S$ the Artin-Burau generators of $B_{n}$. Observe that generators $\sigma_{i}$ are omitted as well as the trivial group relations on them. With the above notation Markov [Mar45] used $s_{i, i+1} \sigma_{i}^{-1}$ to replace $\sigma_{i}$, and $\sigma_{i}^{-2}=s_{i, i+1}^{-1}$ to replace $\sigma_{i}^{-1} \sigma_{i}=1$.

Then we order the set $S$ in the following way:

$$
S_{n}<S_{n-1}<\cdots<S_{2}<\Sigma^{-1}
$$

Clearly, in the above chain, any letter of $S_{n}$ is less than any letter of $S_{n-1}$ and so on. Also we define for each $j$

$$
s_{1, j}^{-1}<s_{1, j}<s_{2, j}^{-1}<\cdots<s_{j-1, j}, \quad \text { and } \quad \sigma_{1}^{-1}<\sigma_{2}^{-1}<\ldots \sigma_{n-1}^{-1} .
$$

With above notation, we now able to order the $S$-words by using the inverse tower order, according to the fixed presentation of $S$ as the union of $S_{j}$ and $\Sigma^{-1}$. We order the $S_{n}$-words by the deg-inlex order, i.e., we first compare the words by length and than by inverse lexicographical order, starting from their last letters.

The following abbreviations are taken from Mar45.

$$
\sigma_{i, j+1}=\sigma_{i}^{-1} \ldots \sigma_{j}^{-1}, 1 \leq i \leq j \leq n-1, \sigma_{i i}=1 .
$$

Also we denote $\{a, b\}=b^{-1} a b$.

\section{MAin RESUlts}

We first cite some crucial results from [Mar45, Ar47.

The first lemma is fairly easy. 
Lemma 3.1. ( Mar45, Lemma 3], Ar47, p.119]) The following relations hold in the braid group $B_{n}$ for $(\delta= \pm 1)$ :

$$
\begin{aligned}
& \sigma_{k}^{-1} s_{i, j}^{\delta}=s_{i, j}^{\delta} \sigma_{k}^{-1}, \quad k \neq i-1, i, j-1, j, \\
& \sigma_{i}^{-1} s_{i, i+1}^{\delta}=s_{i, i+1}^{\delta} \sigma_{i}^{-1} \\
& \sigma_{i-1}^{-1} s_{i, j}^{\delta}=s_{i-1, j}^{\delta} \sigma_{i-1}^{-1}, \\
& \sigma_{i}^{-1} s_{i, j}^{\delta}=\left\{s_{i+1, j}^{\delta}, s_{i, i+1}\right\} \sigma_{i}^{-1}, \\
& \sigma_{j-1}^{-1} s_{i, j}^{\delta}=s_{i, j-1}^{\delta} \sigma_{j-1}^{-1}, \\
& \sigma_{j}^{-1} s_{i, j}^{\delta}=\left\{s_{i, j+1}^{\delta}, s_{j, j+1}\right\} \sigma_{j}^{-1} .
\end{aligned}
$$

Also we have

$$
\begin{aligned}
& \sigma_{i-1} s_{i, j}^{\delta}=\left\{s_{i-1, j}^{\delta}, s_{i-1, i}^{-1}\right\} \sigma_{i-1}, \\
& \sigma_{i} s_{i, j}^{\delta}=s_{i+1, j}^{\delta} \sigma_{i}, \\
& \sigma_{j-1} s_{i, j}^{\delta}=\left\{s_{i, j-1}^{\delta}, s_{j-1, j}^{-1}\right\} \sigma_{j-1}, \\
& \sigma_{j} s_{i, j}^{\delta}=s_{i, j+1}^{\delta} \sigma_{j} .
\end{aligned}
$$

Remark 3.2. We note that last relations from the above lemma will not in Gröbner-Shirshov basis of the braid group $B_{n}$ (see below). We use them for a sketch of a proof of next Lemma 3.3 .

Lemma 3.3. ([Mar45, Lemmas 6 and 7], [Ar47, Theorem 18]) The following relations hold in the braid group $B_{n}$ for all $i<j<k<l$, $\varepsilon= \pm 1$ :

$$
\begin{aligned}
s_{j, k}^{-1} s_{k, l}^{\varepsilon} & =\left\{s_{k, l}^{\varepsilon}, s_{j, l}^{-1}\right\} s_{j, k}^{-1}, \\
s_{j, k} s_{k, l}^{\varepsilon} & =\left\{s_{k, l}^{\varepsilon}, s_{j, l} s_{k, l}\right\} s_{j, k}, \\
s_{j, k}^{-1} s_{j, l}^{\varepsilon} & =\left\{s_{j, l}^{\varepsilon}, s_{k, l}^{-1} s_{j, l}^{-1}\right\} s_{j, k}^{-1}, \\
s_{j, k} s_{j, l}^{\varepsilon} & =\left\{s_{j, l}^{\varepsilon}, s_{k, l}\right\} s_{j, k}, \\
s_{i, k}^{-1} s_{j, l}^{\varepsilon} & =\left\{s_{j, l}^{\varepsilon}, s_{k, l} s_{i, l} s_{k, l}^{-1} s_{i, l}^{-1}\right\} s_{i, k}^{-1}, \\
s_{i, k} s_{j, l}^{\varepsilon} & =\left\{s_{j, l}^{\varepsilon}, s_{i, l}^{-1} s_{k, l}^{-1} s_{i, l} s_{k, l}\right\} s_{i, k} .
\end{aligned}
$$

Also for $j<i<k<l$ or $i<k<j<l$, and $\varepsilon, \delta= \pm 1$

$$
s_{i, k}^{\delta} s_{j, l}^{\varepsilon}=s_{j, l}^{\varepsilon} s_{i, k}^{\delta} .
$$

Proof. We provide a proof of (3.7) for $\varepsilon=1$ as a typical example. We use arguments given by Markov in [Mar45]. First we can easily see that the relation holds for $j=k-1, l=k+1$. We assume that (3.7) holds for $j<k<l$. Then we prove it for $j-1$ and $l+1$ using Lemma 3.1. We deduce the following equalities by direct computation:

$$
\begin{aligned}
& s_{j, k}^{-1} s_{k, l+1}=s_{j, k}^{-1}\left\{s_{k, l}, \sigma_{l}^{-1}\right\}=\left\{\left\{s_{k, l}, s_{j, l}^{-1}\right\}, \sigma_{l}^{-1}\right\} s_{j, k}^{-1}=\left\{s_{k, l+1}, s_{j, l+1}^{-1}\right\} s_{j, k}^{-1}, \\
& s_{j-1, k}^{-1} s_{k, l}=\left\{s_{j, k}^{-1}, \sigma_{j-1}\right\} s_{k, l}=\sigma_{j-1}^{-1} s_{j, k}^{-1} \sigma_{j-1} s_{k, l}=\sigma_{j-1}^{-1} s_{j, k}^{-1} s_{k, l} \sigma_{j-1}= \\
& \left\{\left\{s_{k, l}, s_{j, l}^{-1}\right\} s_{j, k}^{-1}, \sigma_{j-1}\right\}=\left\{s_{k, l}, s_{j-1, l}^{-1}\right\} s_{j-1, k}^{-1} .
\end{aligned}
$$


This shows that (3.7) holds.

Finally, we have the following relations in the braid group $B_{n}$ ( see [Mar45, Lemma 5]). A proof is fairly simple.

Lemma 3.4. The following relations hold in the braid group $B_{n}$ :

$$
\begin{aligned}
& \sigma_{j}^{-1} \sigma_{k}^{-1}=\sigma_{k}^{-1} \sigma_{j}^{-1}, \quad j<k-1, \\
& \sigma_{j, j+1} \sigma_{k, j+1}=\sigma_{k, j+1} \sigma_{j-1, j}, \quad k<j, \\
& \sigma_{i}^{-2}=s_{i, i+1}^{-1},
\end{aligned}
$$

We now call the relations (3.1)-(3.16) together with the trivial relations

$$
s_{i, j}^{ \pm 1} s_{i, j}^{\mp 1}=1
$$

the Artin-Markov relations $\boldsymbol{S}$ for the braid group $B_{n}$ in terms of the Artin-Burau generators.

Using the above relations $\boldsymbol{S}$ together with the definition $\sigma_{i}=s_{i i+1} \sigma_{i}^{-1}$, we can deduce Artin's relations for $B_{n}$.

Namely, in relation (3.15) we let $k=j-1$. Then we have

$$
\sigma_{j}^{-1} \sigma_{j-1}^{-1} \sigma_{j}^{-1}=\sigma_{j-1}^{-1} \sigma_{j}^{-1} \sigma_{j-1}^{-1} .
$$

Also

$$
\sigma_{i}^{-1} \sigma_{i}=\sigma_{i}^{-1} s_{i, i+1} \sigma_{i}^{-1}=s_{i, i+1} \sigma_{i}^{-2}=s_{i, i+1} s_{i, i+1}^{-1}=1,
$$

and the same for $\sigma_{i} \sigma_{i}^{-1}=1$.

Corollary 3.5. The following relations are deduced by the ELW of $\boldsymbol{S}$ (to be more precis, by the ELW of (3.7) - (3.16)):

$$
\begin{aligned}
\sigma_{i, j} \sigma_{k, j} & =\sigma_{k, j} \sigma_{i-1, j-1}, \quad k<i, \\
\sigma_{i, j} \sigma_{k, j} & =s_{i, k+1}^{-1} \sigma_{k+1, j} \sigma_{i, j-1}, \quad i \leq k .
\end{aligned}
$$

\section{Main Theorem}

Using the Artin-Markov relations given in the Section 3, we establish the following theorem.

Theorem 4.1. The Artin-Markov relations form a minimal GröbnerShirshov basis of the braid group $B_{n}$ in term of the Artin-Burau generators relative to the inverse tower order of words.

Proof. There are no inclusion compositions of defining relations. We only need to check all possible intersection compositions. Let us do some for examples.

Let us check a composition of intersection of two relations $f, g$ of the form (3.8) relative to the ambiguity

$$
w=(i j)(j k)(k l), \quad i<j<k<l,
$$

where $(i j)=s_{i, j}$. We have

$f=(i j)(j k)-\{(j k),(i k)(j k)\}(i j), g=(j k)(k l)-\{(k l),(j l)(k l)\}(j k)$. 
We need to prove that

$$
\{(j k),(i k)(j k)\}(i j)(k l) \equiv(i j)\{(k l),(j l)(k l)\}(j k) \bmod (\boldsymbol{S}, w) .
$$

In fact, by computation we deduce that

$(i j)\{(k l),(j l)(k l)\}(j k) \equiv\{(k l),\{(j l),(i l)(j l)\}(k l)\}(i j)(j k) \equiv$

$$
\{(k l),\{(j l),(i l)(j l)\}(k l)\}\{(j k),(i k)(j k)\}(i j) .
$$

For the left hand side of (4.1) we have

$$
\begin{aligned}
& \{(j k),(i k)(j k)\}(i j)(k l) \equiv\{(j k),(i k)(j k)\}(k l)(i j) \equiv \\
& (j k)^{-1}(i k)^{-1}(j k)(i k)(j k)(k l)(i j) \equiv \\
& (j k)^{-1}(i k)^{-1}(j k)(i k)\{(k l),(j l)(k l)\}(j k)(i j) \equiv \\
& (j k)^{-1}(i k)^{-1}(j k)\left\{\{(k l),(i l)(k l)\},\left\{(j l),(i l)^{-1}(k l)^{-1}(i l)(k l)\right\}\right. \\
& \{(k l),(i l)(k l)\}\}(i k)(j k)(i j) \equiv(j k)^{-1}(i k)^{-1}(j k)\{(k l), \\
& \left.\left\{(j l),(i l)^{-1}(k l)^{-1}\right\}(k l)(i l)(k l)\right\}(i k)(j k)(i j) \equiv \\
& (j k)^{-1}(i k)^{-1}(j k)\{(k l),(i l)(j l)(k l)\}(i k)(j k)(i j) \equiv \\
& (j k)^{-1}(i k)^{-1}\{\{(k l),(j l)(k l)\},(i l)\{(j l),(k l)\}\{(k l),(j l)(k l)\}\}(j k)(i k)(j k)(i j) \equiv \\
& (j k)^{-1}\left\{\left\{\left\{(k l),(i l)^{-1}\right\}, X\right\},\left\{(i l),(k l)^{-1}(i l)^{-1}\right\} X\right\}(i k)^{-1}(j k)(i k)(j k)(i j) \equiv \\
& \left(X=\left\{(j l),(k l)(i l)(k l)^{-1}(i l)^{-1}\right\}\left\{(k l),(i l)^{-1}\right\}=\left\{(k l),(i l)^{-1}\right\}\{(j l),(k l)\}\right) \\
& (j k)^{-1}\left\{\left\{\left\{(k l),(i l)^{-1}\right\},\{(j l),(k l)\}\right\},(i l)(j l)(k l)\right\}(i k)^{-1}(j k)(i k)(j k)(i j) \equiv \\
& \left\{\left\{\left\{\left\{(k l),(j l)^{-1}\right\},(i l)^{-1}\right\},\left\{\left\{(j l),(k l)^{-1}(j l)^{-1}\right\},\left\{(k l),(j l)^{-1}\right\}\right\}\right\},\right. \\
& \left.(i l)\left\{(j l),(k l)^{-1}(j l)^{-1}\right\}\left\{(k l),(j l)^{-1}\right\}\right\}(j k)^{-1}(i k)^{-1}(j k)(i k)(j k)(i j)= \\
& (4.3) \quad\left\{\left\{\left\{(k l),(j l)^{-1}(i l)^{-1}\right\},(j l)\right\},(i l)(j l)(k l)\right\}\{(j k),(i k)(j k)\}(i j) .
\end{aligned}
$$

Words (4.2) and (4.3) are the same for

$$
\{(k l),\{(j l),(i l)(j l)\}(k l)\}=\left\{\left\{\left\{(k l),(j l)^{-1}(i l)^{-1}\right\},(j l)\right\},(i l)(j l)(k l)\right\} .
$$

Thus, (4.1) is verified and the composition is checked.

To check the compositions of relations $f, g$ of the forms (3.12), (3.2) respectively, we let $w=\sigma_{q}^{-1}(j k)(k l)$ and let

$$
\begin{aligned}
& f=\sigma_{q}^{-1} s_{j k}-s_{j k} \sigma_{q}^{-1}, \quad q \neq j-1, j, k-1, k, \\
& g=(j k)(k l)-\{(k l),(j l)(k l)\}(j k) .
\end{aligned}
$$

Then we have

$$
(f, g)_{w}=\sigma_{q}^{-1}\{(k l),(j l)(k l)\}(j k)-(j k) \sigma_{q}^{-1}(k l) .
$$


If $q \neq l-1, l$ then it is clear that the composition is trivial. So, we need to consider the following cases:

a) $q=l$. We have

$$
\begin{aligned}
& \sigma_{l}^{-1}\{(k l),(j l)(k l)\}(j k) \equiv\{\{(k, l+1),(l, l+1)\},\{(j, l+1),(l, l+1)\} \\
& \{(k, l+1),(l, l+1)\}\}(j k) \sigma_{l}^{-1} \equiv\{(k, l+1),(j, l+1)(k, l+1)(l, l+1)\}(j k) \sigma_{l}^{-1}, \\
& (j k) \sigma_{l}^{-1}(k l) \equiv(j k)\{(k, l+1),(l, l+1)\} \sigma_{l}^{-1} \\
& \quad \equiv\{(k, l+1),(j, l+1)(k, l+1)(l, l+1)\}(j k) \sigma_{l}^{-1} .
\end{aligned}
$$

Hence, the case is verified.

b) $q=l-1$. We have

$$
\begin{aligned}
& \sigma_{l-1}^{-1}\{(k l),(j l)(k l)\}(j k) \equiv\left\{\{(k, l-1),(j, l-1)(k, l-1)\}(j k) \sigma_{l-1}^{-1},\right. \\
& (j k) \sigma_{l-1}^{-1}(k l) \equiv(j k)(k l) \sigma_{l-1}^{-1} \equiv\{(k, l-1),(j, l-1)(k, l-1)\}(j k) \sigma_{l-1}^{-1} .
\end{aligned}
$$

Hence, the case is also verified.

Finally, we need to check the composition of relations (3.16). We first let

$$
\begin{aligned}
f & =\sigma_{j}^{-1} \sigma_{k}^{-1} \ldots \sigma_{j}^{-1}-\sigma_{k}^{-1} \ldots \sigma_{j}^{-1} \sigma_{j-1}^{-1}, \quad k<j, \\
g & =\sigma_{j}^{-1} \sigma_{l}^{-1} \ldots \sigma_{j}^{-1}-\sigma_{l}^{-1} \ldots \sigma_{j}^{-1} \sigma_{j-1}^{-1}, \quad l<j, \\
\text { and let } w & =\sigma_{j}^{-1} \sigma_{k}^{-1} \ldots \sigma_{j}^{-1} \sigma_{l}^{-1} \ldots \sigma_{j}^{-1} .
\end{aligned}
$$

Then, we have

$$
(f, g)_{w}=-\sigma_{k}^{-1} \ldots \sigma_{j}^{-1} \sigma_{j-1}^{-1} \sigma_{l}^{-1} \ldots \sigma_{j}^{-1}+\sigma_{j}^{-1} \sigma_{k}^{-1} \ldots \sigma_{j-1}^{-1} \sigma_{l}^{-1} \ldots \sigma_{j}^{-1} \sigma_{j-1}^{-1},
$$

We consider the following cases:

a) $l=j-1$. In this case, by Corollary 3.5, we have

$$
\begin{aligned}
& (f, g)_{w} \equiv-\sigma_{k}^{-1} \ldots \sigma_{j}^{-1} s_{j-1, j}^{-1} \sigma_{j}^{-1}+\sigma_{j}^{-1} \sigma_{k}^{-1} \ldots \sigma_{j-2}^{-1} s_{j-1 j}^{-1} \sigma_{j}^{-1} \sigma_{j-1}^{-1} ; \\
& \sigma_{k}^{-1} \ldots \sigma_{j}^{-1} s_{j-1, j}^{-1} \sigma_{j}^{-1} \equiv s_{j, j+1}^{-1} \sigma_{k}^{-1} \ldots \sigma_{j-1}^{-1} s_{j j+1}^{-1} \equiv s_{j, j+1}^{-1} s_{k j+1}^{-1} \sigma_{k}^{-1} \ldots \sigma_{j-1}^{-1} ; \\
& \sigma_{j}^{-1} \sigma_{k}^{-1} \ldots \sigma_{j-2}^{-1} s_{j-1 j}^{-1} \sigma_{j}^{-1} \sigma_{j-1}^{-1} \equiv \sigma_{j}^{-1} s_{k j}^{-1} \sigma_{k}^{-1} \ldots \sigma_{j-2}^{-1} \sigma_{j}^{-1} \sigma_{j-1}^{-1} \\
& \quad \equiv\left\{s_{k j+1}^{-1}, s_{j j+1}\right\} s_{j j+1}^{-1} \sigma_{k}^{-1} \ldots \sigma_{j-1}^{-1} \equiv s_{j, j+1}^{-1} s_{k j+1}^{-1} \sigma_{k}^{-1} \ldots \sigma_{j-1}^{-1},
\end{aligned}
$$

and the case is done.

b) $l<j-1$. We use again Corollary 3.5 to obtain

$$
\begin{aligned}
& \sigma_{k}^{-1} \ldots \sigma_{j}^{-1} \sigma_{j-1}^{-1} \sigma_{l}^{-1} \ldots \sigma_{j}^{-1} \equiv \sigma_{k}^{-1} \ldots \sigma_{j}^{-1} \sigma_{l}^{-1} \ldots \sigma_{j}^{-1} \sigma_{j-2}^{-1} \equiv \sigma_{k j+1} \sigma_{l j+1} \sigma_{j-2}^{-1} \\
& \equiv \sigma_{l j+1} \sigma_{k-1 j} \sigma_{j-2}^{-1} \quad(\text { when } l<k), \text { or } \equiv s_{k l+1}^{-1} \sigma_{l+1 j+1} \sigma_{k j} \sigma_{j-2}^{-1} \quad(\text { when } k \leq l) .
\end{aligned}
$$

If $l<k$, then

$$
\begin{aligned}
\sigma_{j}^{-1} \sigma_{k}^{-1} & \ldots \sigma_{j-1}^{-1} \sigma_{l}^{-1} \ldots \sigma_{j}^{-1} \sigma_{j-1}^{-1} \equiv \sigma_{j}^{-1} \sigma_{k j} \sigma_{l j} \sigma_{j}^{-1} \sigma_{j-1}^{-1} \\
& \equiv \sigma_{j}^{-1} \sigma_{l j} \sigma_{k-1 j-1} \sigma_{j}^{-1} \sigma_{j-1}^{-1} \equiv \sigma_{j}^{-1} \sigma_{l j+1} \sigma_{k-1 j} \equiv \sigma_{l j+1} \sigma_{k-1 j} \sigma_{j-2}^{-1},
\end{aligned}
$$


and we are done. If $l \geq k$, then $k \leq l<j-1$ and so we have

$$
\begin{aligned}
\sigma_{j}^{-1} \sigma_{k}^{-1} \ldots \sigma_{j-1}^{-1} \sigma_{l}^{-1} \ldots \sigma_{j}^{-1} \sigma_{j-1}^{-1} \equiv \sigma_{j}^{-1} \sigma_{k j} \sigma_{l j} \sigma_{j}^{-1} \sigma_{j-1}^{-1} \\
\\
\equiv \sigma_{j}^{-1} s_{k l+1}^{-1} \sigma_{l+1 j} \sigma_{k j-1} \sigma_{j}^{-1} \sigma_{j-1}^{-1} \equiv \sigma_{j}^{-1} s_{k l+1}^{-1} \sigma_{l+1 j+1} \sigma_{k j}
\end{aligned}
$$

and

$$
\sigma_{j}^{-1} s_{k l+1}^{-1} \sigma_{l+1 j+1} \sigma_{k j} \equiv s_{k l+1}^{-1} \sigma_{l+1 j+1} \sigma_{k j} \sigma_{j-2}^{-1},
$$

as desired.

Applying Composition-Diamond Lemma we obtain:

Corollary 4.2. The set of $\boldsymbol{S}$-irreducible words of $B_{n}$ corresponding to the above Gröbner-Shirshov basis $\boldsymbol{S}$ consists of the words

$$
f_{n} f_{n-1} \ldots f_{2} \sigma_{i_{n} n} \sigma_{i_{n-1} n-1} \ldots \sigma_{i_{2} 2}
$$

where $f_{j}$ are free irreducible words in $\left\{s_{i j} \mid i<j\right\}, 2 \leq j \leq n$.

Corollary 4.3 (Markov-Ivanovsky [Mar45, Theorem 6] and Artin [Ar47, Theorem 17 and remark of Theorem 18]). Every word of $B_{n}$ has a unique presentation in the form (4.4).

Let $\Sigma_{n}$ be the symmetric group, i.e., $\Sigma_{n}=\left\langle s_{1}, \ldots, s_{n-1} \mid s_{i}^{2}=1, s_{i+1} s_{i} s_{i+1}=s_{i} s_{i+1} s_{i}, s_{i} s_{j}=s_{j} s_{i}, i-j>1\right\rangle$, and let

$$
S_{i, i}=1 \text { and } S_{i, j+1}=s_{i} s_{i+1} \ldots s_{j}, \quad i<j .
$$

The following lemma was proved in Mar45, Theorem 4, Corollary 6]. It also follows from the fact that

$$
\left\{s_{i}^{2}=1, s_{i} s_{j}=s_{j} s_{i}, i-j>1, S_{j, j+1} S_{k, j+1}=S_{k, j+1} S_{j-1, j}, k<j\right\}
$$

is a Gröbner-Shirshov basis of $\Sigma_{n}$ under the deg-inlex order of words in $\left\{s_{i}\right\}$ (see [BoS01]).

Lemma 4.4. Every element of $\Sigma_{n}$ has a unique presentation in a form

$$
S_{i_{n}, n} S_{i_{n-1}, n-1} \ldots S_{i_{2}, 2}
$$

where $i_{j} \leq j$ and $2 \leq j \leq n$.

Let $P_{n}$ be the group of pure braids. This is the kernel of the natural homomorphism of $B_{n}$ onto $\Sigma_{n}$. From Theorem 4.1, Corollary 4.3 and Lemma 4.4 it follows

Corollary 4.5 (Markov-Ivanovsky [Mar45, Theorem 8] and Artin [Ar47, Theorem 18]). $P_{n}$ is a group with generators $\left\{s_{i j}\right\}$ and defining relations (3.7)-3.13) (which, together with the trivial relations, form a minimal Gröbner-Shirshov basis of $P_{n}$ relative the inverse tower order of words in the generators). 


\section{REFERENCES}

[Ar25] E. Artin, Theory der Zöpfe. Abh. Math. Seminar., Hamburg Univ. 4 (1925), $47-72$.

[Ar47] E. Artin, Theory of braids. Ann. of Math., 48 (1947), 101-126.

[Be78] G. M. Bergman, The diamond lemma for ring theory. Adv. in Math. 29 (1978), 178-218.

[Bo72] L. A. Bokut, Unsolvability of the word problem, and subalgebras of finitely presented Lie algebras. Izv. Akad. Nauk SSSR Ser. Mat. 36 (1972), 1173-1219.

[Bo76] L. A. Bokut, Imbeddings into simple associative algebras. Algebra i Logika 15 (1976), 117-142, 245

[BoS01] L.A. Bokut and L.-S. Shiao, On Gröbner-Shirshov basis of Coxeter groups, Commun. in Algebra 29 (2001), no. 9, 4305-4319.

[Bu65] B. Buchberger. An algorithm for finding a basis for the residue class ring of a zero-dimensional polynomial ideal. (German). Ph. D. thesis, University of Insbruck, Austria, 1965.

[Bu70] B. Buchberger, An algorithmical criteria for the solvability of algebraic systems of equations. (German). Aequationes Math. 4 (1970), 374-383.

[Bur32] W. Burau, Über Zopfinvarianten . Abh. Math. Sem. Hamburg Univ. 9 (1932), 2, 117-124.

[Mar45] A.A. Markov, Foundations of the algebraic theory of braids. Proceedings of the Steklov Mathematical Institute. 16 Moscow-Leningrad, 1945.

[Ne42] M. H. A. Newman, On theories with a combinatorial definition of "equivalence." Ann. of Math. 43 (1942), 223-243.

[Sh62] A. I. Shirshov, Some algorithm problems for Lie algebras. (Russian) Sibirsk. Mat. Z. 3 (1962), 292-296. (Translation appears in ACM SIGSAM Bull. 33 (1999).)

Sobolev Institute of Mathematics, Novosibirsk 630090, Russia

E-mail address: bokut@math.nsc.ru

Faculty of mathematics and mechanics, Novosibirsk State UniverSiTY, Novosibirsk, 630090, Russia

E-mail address: vvch@math.nsc.ru

Faculty of Science, The Chiness University of Hong Kong, Hong Kong, China (SAR)

E-mail address: kpshum@math.cuhk.edu.hk 